\title{
Compositional Analysis of Atherosclerotic Lesions in a Mouse Model: Validation of a New Method Using Brightfield, Fluorescence and Polarized Light Microscopy in Conjunction with Computer-Assisted Image Analysis
}

\author{
M. Wadsworth*, D. Schneider**, B. Sobel**, and D. Taatjes* \\ * Dept.of Pathology and Microscopy Imaging Center, University of Vermont, Burlington, VT 05405 \\ ** Dept. of Medicine, University of Vermont, Burlington, VT 05405
}

An emerging concept in the pathology of unstable atherosclerotic plaques indicates that the plaque composition may be of paramount importance in determining its vulnerability to rupture with consequent clinical sequelae [1]. We previously developed methods for the semi-quantitative analysis of plaque composition in an $\mathrm{ApoE}^{-/}$knockout mouse animal model [2]. We now report further modifications of these methods, and demonstrate their use in assessing plaque composition in different aged ApoE ${ }^{-1}$ knockout mice and their control C57Bl6 counterparts. Hearts were harvested, fixed and frozen from male and female mice in both groups at 10 and 20 weeks of age, following feeding with a high fat diet since weaning at three weeks. Orientation and sectioning of the heart tissue with a cryostat to encompass a $300 \mu \mathrm{m}$ area of the aorta from the sinus region to the valve cusps were performed as previously described [2,3]. For this study, the following constituents of the plaque were assessed: (1) lipid, stained with oil red $\mathrm{O}$ and imaged by brightfield light microscopy; (2) collagen, stained with picrosirius red and imaged by polarized light microscopy; and (3) total cellularity, nuclei stained with Sytox green and imaged by epifluorescence microscopy. Digital images were imported into Universal Imaging MetaMorph software for image analysis.

After 7 or 17 weeks on a high fat diet, the C57B16 animals showed essentially no atherosclerotic plaques in the aortic area analyzed. Thus, the lesion composition was compared in the 10 week $(\mathrm{N}=3)$ and 20 week old $(\mathrm{N}=4) \mathrm{ApoE}^{-/}$knockout mice. Although the average per cent of the vessel occupied by lesion increased 1.5 fold from the 10 to 20 week old animals, the per cent composition attributed to lipid (Figure 1), and cellularity (Figure 2) did not appear to change significantly in this limited number of animals. However, the per cent composition attributed to collagen showed a 2.5 fold increase in the 20 week old female animals only (Figure 3). The results of all of the analyses are presented in the graph in Figure 4.

The results and analysis of the data presented in this study demonstrate the utility of these new combined methods in elucidating the composition of atherosclerotic plaques in a mouse model. Although as anticipated, the per cent lipid and cellular composition of plaques did not appear to change significantly with age, the increased expression of collagen with age in the female mice was unexpected, and will need to be validated by further analysis of more animals. With knowledge of the baseline plaque composition in $\mathrm{ApoE}^{-/}$knockout mice, we are now examin ing the effect of overand under expression of plasminogen activator inhibitor 1 (PAI -1) on plaque composition in (PAI-1) transgenic mice bred on an $\mathrm{ApoE}^{-/}$background.

\section{References}

[1] B.E. Sobel, Circulation 99 (1999) 2496.

[2] D.J. Taatjes et al., Histochem.Cell Biol. 113 (2000) 161.

[3] B. Paigen et al., Atherosclerosis 68 (1987) 231. 


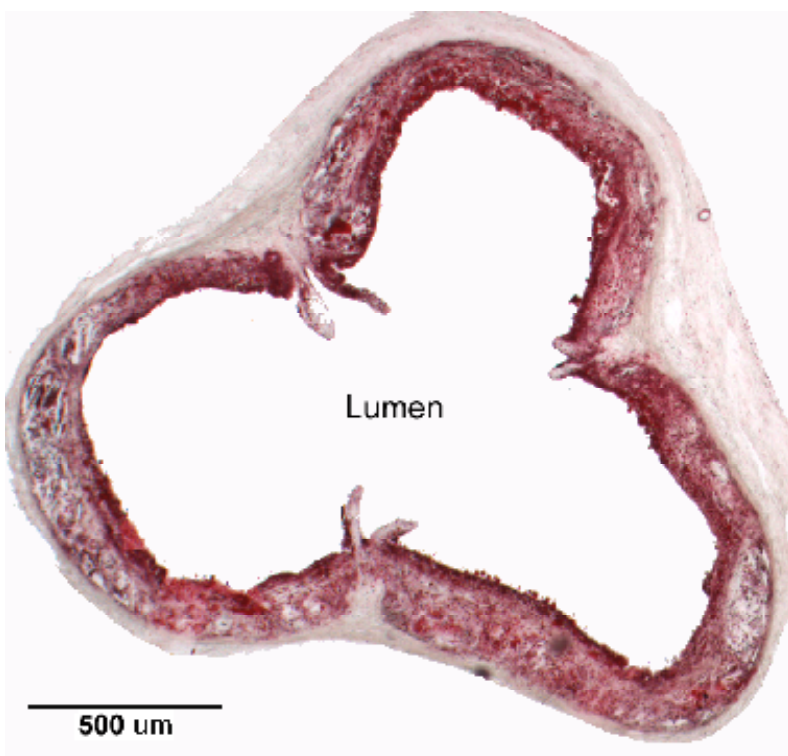

Fig.1 Brightfield image of cryosection from mouse aorta stained with oil-red $\mathrm{O}$ demonstrating lipid.

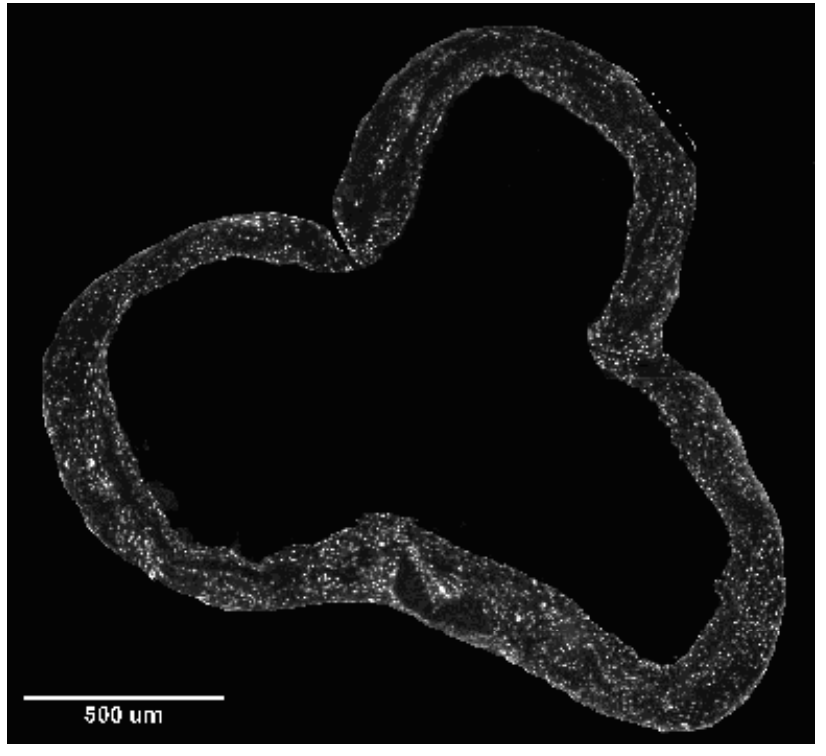

Fig. 2 Epi-fluorescence image of an adjacent section from mouse aortic lesion stained with Sytox green demonstrating cellularity.

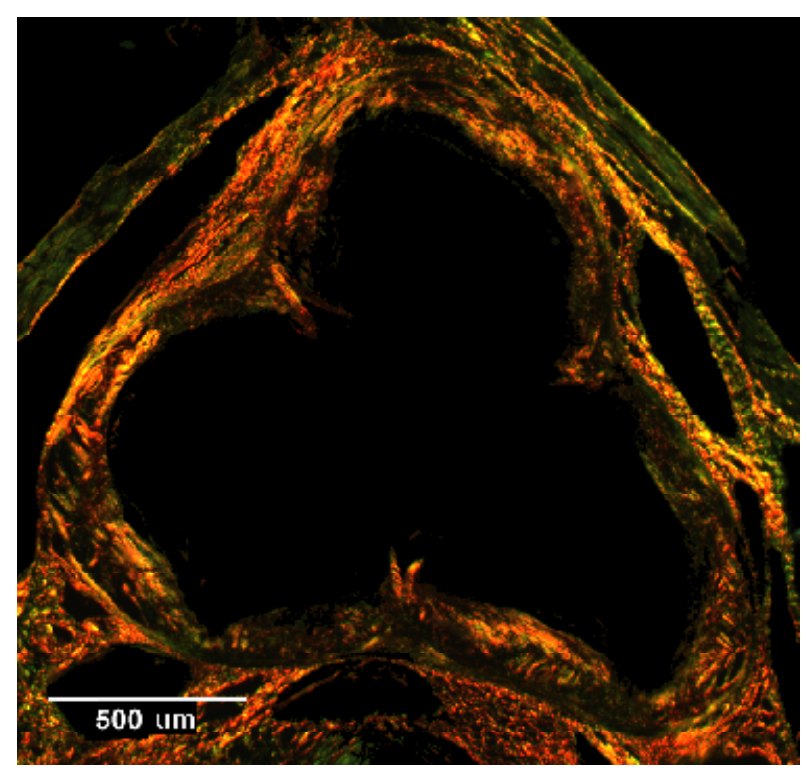

Fig. 3 Polarized light microscopy image of an adjacent section from mouse aorta demonstrating collagen.

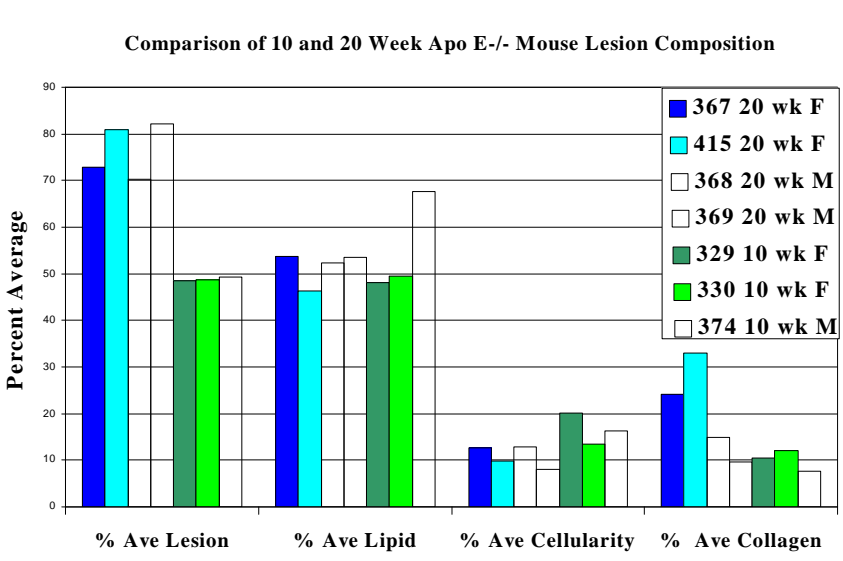

Fig. 4 Summary of comparison of lesion composition in 10 and 20 week old mice. 